\title{
Intersubject Variability of and Genetic Effects on the Brain's Functional Connectivity during Infancy
}

\author{
Wei Gao, ${ }^{1,2}$ Amanda Elton, ${ }^{1,2}$ Hongtu Zhu, ${ }^{2,3}$ Sarael Alcauter, ${ }^{1,2}$ 이. Keith Smith, ${ }^{4}$ John H. Gilmore, ${ }^{5}$ and Weili Lin ${ }^{1,2}$ \\ Departments of ${ }^{1}$ Radiology, ${ }^{2}$ Biomedical Research Imaging Center, ${ }^{3}$ Biostatistics, ${ }^{4}$ Radiology, and ${ }^{5}$ Psychiatry, University of North Carolina at Chapel Hill, \\ Chapel Hill, North Carolina 27599
}

\begin{abstract}
Infancy is a period featuring a high level of intersubject variability but the brain basis for such variability and the potential genetic/ environmental contributions remain largely unexplored. The assessment of the brain's functional connectivity during infancy by the resting state functional magnetic resonance imaging (rsfMRI) technique (Biswal et al., 1995) provides a unique means to probe the brain basis of intersubject variability during infancy. In this study, an unusually large typically developing human infant sample including 58 singletons, 132 dizygotic twins, and 98 monozygotic twins with rsfMRI scans during the first 2 years of life was recruited to delineate the spatial and temporal developmental patterns of both the intersubject variability of and genetic effects on the brain's functional connectivity. Through systematic voxelwise functional connectivity analyses, our results revealed that the intersubject variability at birth features lower variability in primary functional areas but higher values in association areas. Although the relative pattern remains largely consistent, the magnitude of intersubject variability undergoes an interesting U-shaped growth during the first 2 years of life. Overall, the intersubject variability patterns during infancy show both adult-like and infant-specific characteristics (Mueller et al., 2013). On the other hand, age-dependent genetic effects were observed showing significant but bidirectional relationships with intersubject variability. The temporal and spatial patterns of the intersubject variability of and genetic contributions to the brain's functional connectivity documented in this study shed light on the largely uncharted functional development of the brain during infancy.
\end{abstract}

Key words: early brain development; functional connectivity; genetic effects; infancy; intersubject variability; resting state

\section{Introduction}

Human infancy represents a period featuring a high-level of intersubject variability in behavior but the brain basis for such variability remains largely unexplored (Snyder et al., 2002). The lack of appropriate noninvasive functional brain imaging approaches applicable to normal developing infants may be one of the most important reasons for the scarceness of such studies. By measuring the temporal synchronization of blood oxygen level-dependent signals (BOLD) between different regions of the brain in the absence of any explicit tasks, the resting-state functional magnetic resonance imaging (rsfMRI) (Biswal et al., 1995) technique made the noninvasive evaluation of the brain's functional organization in infants possible (Fransson et al., 2007; Gao et al., 2009a, 2011, 2013; Doria et al., 2010; Smyser et al., 2011). Particularly, the brain's functional organization pattern has been increasingly accepted as the "blueprint" of one's mind and likely dictates one’s behavior (McIntosh, 2000; Seung, 2013). There-

Received Dec. 2, 2013; revised June 11, 2014; accepted July 10, 2014.

Author contributions: W.G., J.H.G., and W.L. designed research; W.G., A.E., J.H.G., and W.L. performed research; W.G., A.E., H.Z., S.A., and J.K.S. analyzed data; W.G., A.E., H.Z., J.H.G., and W.L. wrote the paper.

This work was supported by National Institutes of Health (R01MH070890-09A1 to J.H.G.); Foundation of Hope for Research and Treatment of Mental IIIness Award to W.G., and UNC-Chapel Hill start-up to W.G.

The authors declare no competing financial interests.

Correspondence should be addressed to Dr Wei Gao, University of North Carolina at Chapel Hill, Department of

Radiology and Biomedical Research Imaging Center, Room 3105, Bioinformatics Building, Chapel Hill, NC 27599.

E-mail:wgao@email.unc.edu.

DOI:10.1523/JNEUROSCI.5072-13.2014

Copyright $\odot 2014$ the authors $\quad 0270-6474 / 14 / 3411288-09 \$ 15.00 / 0$ fore, given its relative straightforward application in infant subjects, rsfMRI-derived functional connectivity measure represents a premium means to investigate the brain basis of intersubject variability during infancy. In fact, Mueller et al. (2013) have applied rsfMRI to a group of adult subjects and quantified the intersubject variability of the whole-brain functional connectivity pattern. Their results mainly revealed lower interindividual variability in primary functional areas but higher variability in heteromodal association areas, which is highly consistent with our observation of the behavioral variability pattern (Mueller et al., 2013). Regarding the underlying mechanisms contributing to this biased distribution pattern, they postulated that the prolonged course of maturation and weaker genetic control during development may be major factors potentially contributing to the observed higher intersubject variability within the brain's association areas.

However, there is currently no study on either the intersubject variability of or the genetic effects on the brain's functional connectivity during infancy, leaving the above-mentioned explanations largely invalidated. In this study, a large and unique dataset including both male and female singletons and twins scanned during the first 2 years of life [ 410 datasets in total; neonates: 36 singletons, 80 dizygotic (DZ) twin infants, 62 monozygotic (MZ) twin infants; 1-year-olds: 46 singletons, $50 \mathrm{DZ}$ twin infants, 36 MZ twin infants; 2-year-olds: 26 singletons, 38 DZ twin infants, $36 \mathrm{MZ}$ twin infants] was used to characterize the intersubject variability of and genetic effects on the brain's functional connec- 
Table 1. Detailed demographic information of subjects included in this study

\begin{tabular}{lrll}
\hline & $n$ & $\begin{array}{l}\text { Sex } \\
\text { (males/females) }\end{array}$ & $\begin{array}{l}\text { Age } \\
\text { (postnatal days) }\end{array}$ \\
\hline 1 month & 178 & $129 / 85$ & $37 \pm 18$ \\
$\quad$ Singletons & 36 & $17 / 19$ & $22 \pm 9$ \\
Dizygotic twins & 80 & $46 / 34$ & $36 \pm 15$ \\
Monozygotic twins & 62 & $30 / 32$ & $46 \pm 16$ \\
12 months & 132 & $87 / 59$ & $397 \pm 26$ \\
Singletons & 46 & $23 / 23$ & $378 \pm 17$ \\
Dizygotic twins & 50 & $31 / 19$ & $402 \pm 28$ \\
Monozygotic twins & 36 & $16 / 20$ & $410 \pm 17$ \\
24 months & 100 & $58 / 41$ & $765 \pm 32$ \\
$\quad$ Singletons & 26 & $17 / 9$ & $741 \pm 25$ \\
Dizygotic twins & 38 & $25 / 13$ & $779 \pm 35$ \\
Monozygotic twins & 36 & $16 / 20$ & $766 \pm 23$ \\
\hline
\end{tabular}

tivity during infancy. We aimed to delineate both the spatial distribution and temporal development pattern of these two important functional connectivity properties during the first 2 years of life, and to provide a better understanding of the interplay between genes and intersubject variability with respect to the brain's functional organization during this critical developmental period. Our results provide consistent evidence regarding the role of prolonged maturation in heightened intersubject variability in association areas but largely challenge the previously postulated simplistic relationship between weaker genetic control and higher intersubject variability (Mueller et al., 2013). Additional infant-specific patterns of intersubject variability and genetic effects were delineated and discussed.

\section{Materials and Methods}

Subjects. The subjects in this study were male and female singletons and twins participating in a large study of brain development in normal and high risk children (Gilmore et al., 2007, 2012; Gao et al., 2011, 2014a). Number of subjects, age, and gender information are reported by age group and singleton/twin status in Table 1 . Informed consent was obtained from the parents of all participants and the experimental protocols were approved by the Institutional Review Board, University of North Carolina at Chapel Hill. Subjects who met the inclusion and exclusion criteria were included in this study. Inclusion criteria were birth between the gestational ages of 35 and 42 weeks, weight that was appropriate for gestational age, and the absence of major pregnancy and delivery complications as defined in the exclusion criteria. Exclusion criteria included maternal pre-eclampsia, placental abruption, neonatal hypoxia, or any neonatal illness requiring $>1 \mathrm{~d}$ Neonatal intensive care unit stay, mother with HIV, any mother actively using illegal drugs/narcotics during pregnancy, or any chromosomal or major congenital abnormality.

All subjects were scanned while in a natural sleep state (scans were scheduled to coincide with normal nap times). Images were acquired using a $3 \mathrm{~T}$ MR scanner (Allegra or Trio, Siemens Medical Systems) housed in the Biomedical Research Imaging Center at UNC-Chapel Hill. A 3D MP-RAGE sequence was used to provide anatomical images to coregister all images among subjects. The imaging parameters were as follows: repetition time (TR) $=1820 \mathrm{~ms}$ (Allegra) $/ 1900 \mathrm{~ms}$ (Trio); echo time $(\mathrm{TE})=4.38 \mathrm{~ms}$ (Allegra) $/ 3.74 \mathrm{~ms}$ (Trio); inversion time $=1100 \mathrm{~ms}$; 144 slices; and voxel size $=1 \times 1 \times 1 \mathrm{~mm}^{3}$. For the rs-fMRI, a T2*weighted EPI sequence was used to acquire images. The imaging parameters were as follows: $\mathrm{TR}=2 \mathrm{~s}, \mathrm{TE}=32 \mathrm{~ms} ; 33$ slices; and voxel size $=4 \times$ $4 \times 4 \mathrm{~mm}^{3}$. This sequence was repeated 150 times so as to providing time series images.

Preprocessing. fMRI data preprocessing included removal of the first 10 volumes, slice timing correction, motion correction, spatial smoothing, and temporal filtering between 0.01 and $0.08 \mathrm{~Hz}$. To account for differences in brain sizes across age groups, we used an adaptive approach to spatial smoothing (Gao et al., 2014b), applying a $4 \mathrm{~mm}, 8.25 \mathrm{~mm}$ or 8.93 $\mathrm{mm}$ full-width at half-maximum Gaussian kernel (calculated based on the ratios of brain sizes across different age groups) to data from neonates, 1-year-olds, and 2-year-olds, respectively. Additionally, noise signals extracted from white matter, CSF, and the global signal were regressed out of the time series. To further minimize motion artifacts, we applied a "scrubbing" process in which volumes displaced $>0.2 \mathrm{~mm}$ displacement from the previous volume and for which global signal change between volumes exceeded $0.3 \%$ were removed, in addition to the one volume preceding and two following (Power et al., 2012, 2013). We set up a lower threshold of 75 volumes so that subjects whose data were scrubbed to $<75$ volumes were excluded from subsequent analyses. Finally, data from 178 neonates ( 36 singletons, $40 \mathrm{DZ}$ twin pairs, $31 \mathrm{MZ}$ twin pairs), 132 1-year-olds ( 46 singletons, $25 \mathrm{DZ}$ twin pairs, $18 \mathrm{MZ}$ twin pairs), and 1002 -year-olds (26 singletons, $19 \mathrm{DZ}$ twin pairs, $18 \mathrm{MZ}$ twin pairs) were included. Skull stripping of T1 images was done using a newly developed method named LABEL for neonates (Shi et al., 2012) and BET function in FMRIB Software Library (FSL) for 1- and 2-year-olds. For each subject, after an initial rigid alignment between functional data and the T1 high resolution structural image, a nonlinear transformation field was obtained from the individual T1 image to a longitudinal T1template, i.e., a T1 image of a subject scanned at 2 weeks, 1 year, and 2 years using FSL (Smith et al., 2004; Gao et al., 2010). The combined transformation field was used to warp the preprocessed rsfMRI data to the template.

Following preprocessing, we conducted secondary analyses to test for motion differences between the three age groups using one-way ANOVA. For singleton subjects, which were used to test the effect of development on variability, the number of volumes removed differed for neonates $(27.1 \pm 9.5)$ compared with 1 -year-olds $(21.3 \pm 10.5)$ and 2 -year-olds $(20.3 \pm 11.7)\left(F_{(2,105)}=5.04, p=0.008\right)$. Across the entire sample of singletons and twins, there was an overall effect of age on number of volumes removed $\left(F_{(2,407)}=6.38 ; p=0.002\right)$, where 1-yearolds had less removed volumes $(14.6 \pm 12.6)$ than neonates $(20.9 \pm 17.0)$ or 2 -year-olds $(19.6 \pm 17.4)$. Following the scrubbing procedure, the framewise displacement (FD) present in the remaining volumes was low and did not significantly differ across the three age groups in singletons (neonates: $0.073 \pm 0.011$; 1 -year-olds: $0.075 \pm 0.013$; 2 -year-olds: $\left.0.082 \pm 0.017 ; F_{(2,105)}=2.76 ; p=0.068\right)$. However, there was a significant age effect on framewise displacement across the entire sample where neonates demonstrated significantly lower movement $(0.070 \pm 0.017)$ than the 1 -year-olds $(0.076 \pm 0.014)$ and 2 -year-olds $(0.077 \pm 0.016$; $\left.F_{(2,407)}=7.44 ; p=0.007\right)$. Therefore, due to the potential for age-related differences in motion to influence our findings, we entered these two motion-related measures (i.e., number of volumes removed and residual FD) as covariates of no interest in our statistical models as described below.

Variability in functional connectivity. For each subject, the Pearson correlation of the time series of each voxel within the gray matter mask with every other voxel in the mask was calculated and Fisher $z$-transformed, producing an $n$-voxel $\times n$-voxel correlation matrix for every subject. A set of infant brain structural atlases (Shi et al., 2011) were used to generate gray matter masks for all three age groups. Specifically, the composite mask covering the 90 automated anatomical labeling cortical regions in each of the infant atlases was warped to our age-specific templates and served as the age-specific gray matter mask. For two subjects within a twin pair (denoted as Twin A and Twin B), the variability of voxelwise whole brain connectivity patterns was calculated as one minus the correlation between corresponding columns of the correlation matrix from Twins A and B. For singletons, this same process was conducted on nonsibling pairs where each subject was matched for postnatal age with one other subject, providing 18, 23, and 13 singleton "pairs" for neonates, 1-year-olds, and 2-year-olds respectively. This process provided a means of conducting genetics analyses with minimal influence from variability in age-dependent development (Gao et al., 2014a,b). Age-matching of pairs resulted in average $( \pm S D)$ age differences at the time of the scan of $1.2( \pm 3.4) \mathrm{d}$ for neonates, $2.2( \pm 4.1) \mathrm{d}$ for 1-year-olds, and $3.1( \pm 5.4) \mathrm{d}$ for 2-year-olds. Variability maps were averaged across pairs at each age (neonate, 1 year, 2 years) and each genetic group (singleton, DZ, MZ), providing nine group-mean variability maps. Subsequently, all individual variability maps from the singleton pairs in each 
age group were warped to an MNI template using the 4D HAMMER image registration method (Shen and Davatzikos, 2004) to allow for comparisons across the three age groups to detect significant developmental changes in the intersubject variability of functional connectivity during infancy.

Testing the effect of development on variability. The effect of developmental age on variability in the voxelwise connectivity pattern was calculated using a one-way analysis of covariance with age (neonate, 1 year, 2 years) as a single factor, controlling for within-pair sex differences (i.e., $1=$ male/female pairs, $0=$ male/male or female/female pairs), as well as the within-pair mean number of volumes removed and within-pair mean framewise displacement of retained volumes. Planned comparisons tested the variability in voxel connectivity between neonates and 1 -year-olds, and between 1-year-olds and 2-year-olds for each genetic group (i.e., singletons, DZs, and MZs). Results were corrected for multiple tests (resulting from voxelwise univariate testing) based on Monte Carlo simulations conducted in 3dClustSim in AFNI software (Cox, 1996) by combining a $p$ value threshold of 0.005 and a cluster size threshold of 13 contiguous voxels.

Network-level variability. Canonical brain networks were obtained by downloading the 10 resting-state network components reported by Smith et al. (2009) from the FMRIB website (http://fsl.fmrib.ox.ac. uk/analysis/brainmap + rsns/) and warping to our infant template spaces. Omitting the cerebellar network, the remaining nine components (i.e., sensorimotor, medial occipital, later visual/parietal, occipital pole, default-mode, salience, and bilateral frontoparietal networks) were converted to binary masks using a threshold of $Z>2.0$. The mean value of all voxels within each of the nine networks was calculated based on the group-level variability maps of each age group to represent networkspecific variability. To examine developmental changes of network level variability, the voxelwise values of the group-mean variability within each network were tested across different age groups (i.e., between neonates and 1-year-olds; between 1- and 2-year-olds) using paired $t$ tests.

Relationship of variability to local and distant connectivity. Procedures for calculating local and distant connectivity were the same as those used in by Mueller et al., 2013 and Sepulcre et al., 2010. Calculations were conducted on the mean voxelwise connectivity matrix for each age group. Given the potential effects of smoothing on local connectivity values, all local and distant connectivity measures were calculated using unsmoothed data. In graph analyses, degree is a measure of the number of binary edges connected to a given node, frequently defined using a certain connectivity threshold (i.e., correlation). In our analyses, based on Mueller et al., 2013, degree distant connectivity at a given voxel was defined as the number of voxels located outside of a predefined radius (detailed below) and demonstrating a correlation of $r>0.25$ with that seed voxel. Degree local connectivity was defined as the number of voxels within the local neighborhood (voxels within a predefined radius as detailed below) that demonstrated a correlation of $r>0.25$ with the seed voxel. To account for differences across the brain in the number of gray matter voxels included in the local neighborhood, the local degree value was weighted as a proportion of connections relative to the total number of gray matter voxels within the local sphere (Sepulcre et al., 2010). Percentage distant connectivity was calculated as the degree of distant connections divided by the sum of distant and local connections. For the radius thresholds, we adopted the local and long distance radius thresholds of Mueller et al., 2013, which are 12 and $25 \mathrm{~mm}$ for the local and distant connectivity radius threshold, respectively. However, because we performed these analyses in the age-specific template space, these values were adjusted according to the size of the infant-specific brain template relative to MNI space. Therefore, we calculated the ratio of the longest distance of the age-specific template to the longest distance of the MNI template and multiplied this proportion by 12 and $25 \mathrm{~mm}$ to adjust the local radius (i.e., neonates: $7.6 \mathrm{~mm}$; 1-year-olds: $10.0 \mathrm{~mm}$; 2-year-olds: $10.3 \mathrm{~mm}$ ) and long distance radius (i.e., neonates: $16.0 \mathrm{~mm}$; 1-year-olds: $21.0 \mathrm{~mm}$; 2-year-olds: $21.5 \mathrm{~mm}$ ), respectively, for each age group. Degree local connectivity was converted to Z-scores to standardize the results across age groups. Relationships between voxelwise measures of percentage distant connectivity, degree local connectivity and intersubject variability for each age group was examined using Pearson correlation analyses.
Testing the effect of shared genes on similarity. For neonates, 1-yearolds, and 2-year-olds separately, a voxelwise general linear model (GLM) regression analysis tested the effect of genetic relatedness, modeled as the percentage of shared genes (singletons pairs $=0 \%, \mathrm{DZ}$ pairs $=50 \%$ and $\mathrm{MZ}$ pairs $=100 \%)$ and shared environment (singletons pairs $=0, \mathrm{DZ}$ pairs = 1 and MZ pairs = 1), on similarity (i.e., correlation of the voxelwise functional connectivity patterns within the gray matter mask) of functional connectivity, based on data from all singleton, DZ, and MZ pairs in each age group. We further included within-pair sex differences (i.e., $1=$ male/female pairs, $0=$ male/male or female/female pairs), mean number of volumes removed and mean framewise displacement of retained volumes as covariates of no interest. Each of the three agespecific results was corrected for multiple comparisons using $3 \mathrm{dCl}$ (ustSim ( $p<0.005,13$ voxels). Again, mean $\beta$ values representing the estimates of genetic effects for each age group were extracted from all voxels of each of the nine brain network masks to provide network-level estimates of genetic influence. Similarly, to examine developmental changes of genetic effects, the voxelwise group estimate of genetic effect within each network were tested across different age groups (i.e., between neonates and 1-year-olds; between 1- and 2-year-olds) using paired $t$ tests.

Relationship between genetic effects and variability. To test the linear relationship between genetic influence and variability estimates, we adopted a "searchlight" approach (Kriegeskorte et al., 2006) to calculate the local correlation within 27 -voxel neighborhoods. A searchlight was centered on each voxel and was defined as that voxel and all touching neighbors (side, edge, or corner). A Pearson correlation coefficient was calculated from a vector containing variability values and a vector containing genetic effect estimates for all voxels within the searchlight (27 voxels) and the correlation value was assigned to the center voxel. This processes produced a map of correlation values representing the voxelwise relationship between genetic influence and variability. Results were corrected for multiple tests based on $3 \mathrm{dClust} \operatorname{Sim}(p<0.005,13$ voxels). To ensure our findings were not driven by our choice in neighborhood size, we performed identical analyses with a smaller (7 voxel) and larger (57 voxel) spherical searchlight.

\section{Results}

The intersubject variability of the brain's functional connectivity in singletons during infancy is presented in Figure $1 A$. Strikingly, the relative spatial distributions across the three age groups demonstrated a consistent pattern of lower variability in primary functional areas and higher variability in association areas, suggestive of developmental precursors of the pattern that has been previously observed in adults (Mueller et al., 2013). Specifically, paracentral lobule, pre-/postcentral gyrus, and occipital pole regions were among the areas showing the lowest intersubject variability, whereas the lateral frontal areas consistently showed higher variability. However, developmental improvements showing increasing similarity to the pattern reported in adults were also observed. For example, lower variability in medial visual areas and higher variability in the superior temporal lobes do not become apparent until 1 year of age or later (Fig. 1A). Temporally, a wide-spread decrease in variability was observed during the first year of life whereas a region-specific increase was apparent during the second year (Fig. 1B). When assessed at the network level, the distribution patterns across all three age groups revealed both common and age-specific trends. As a common pattern, the four motor and visual-related networks, including sensorimotor, medial occipital, later visual/parietal, and occipital pole networks showed below-average variability while the three higher-order cognitive networks, including the salience and bilateral frontoparietal networks, generally demonstrated aboveaverage intersubject variability (Fig. $1 C-E$ ). However, there were also developmental changes toward more adult-like patterns: the default-mode network was ranked as the second in neonates but was ranked in the middle ( 5 of 9 networks) in both 1 - and 2-year- 


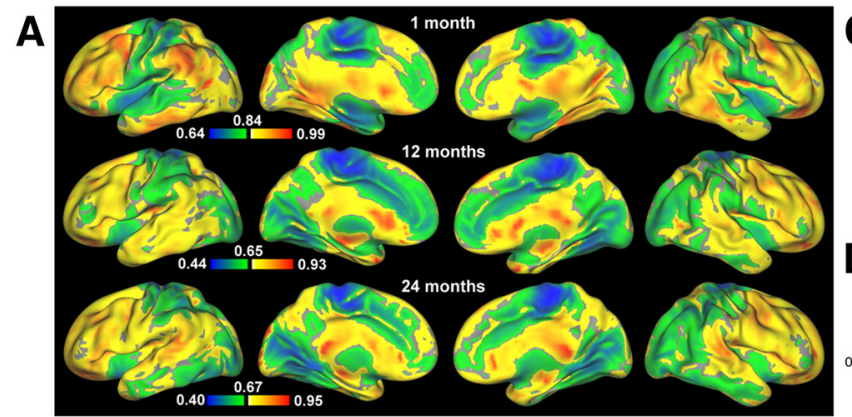

B

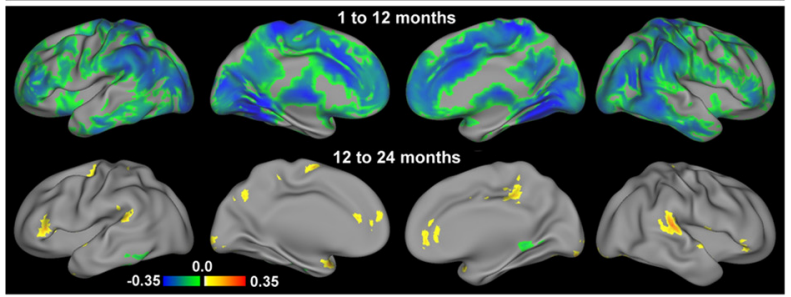

$\mathbf{C}_{0.80}$

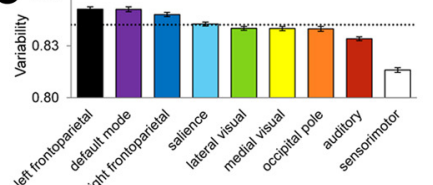

D

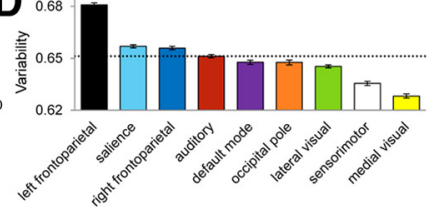

$\mathrm{E}$

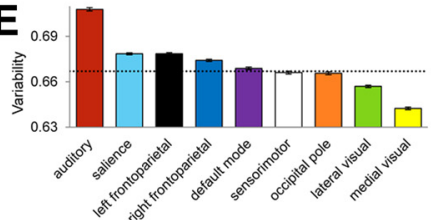

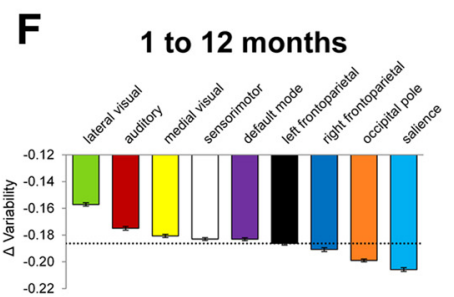

G

12 to 24 months

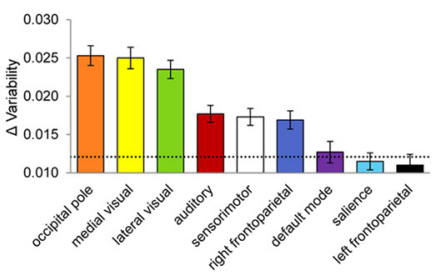

Figure 1. Intersubject variability pattern of the brain's functional connectivity during infancy (singletons). $\boldsymbol{A}$, Intersubject variability map of the brain's functional connectivity in neonates (top row), 1-year-olds (middle row), and 2-year-olds (bottom row); values were demeaned (the mean values are labeled at the middle of the color bars): cool colors represent values below the whole-brain mean intersubject variability and warm colors represent values greater than the mean. $\boldsymbol{B}$, Areas showing statistically significant changes $(p<0.05$ after cluster-size multiplecomparisons correction) in intersubject variability of the brain's functional connectivity during the first year (top row) and second year of life (bottom row). C, Network-level intersubject variability of the brain's functional connectivity at 1 month measured based on singleton subjects. $\boldsymbol{D}$, Network-level intersubject variability of the brain's functional connectivity at 12 months measured based on singleton subjects. $\boldsymbol{E}$, Network-level intersubject variability of the brain's functional connectivity at 24 months measured based on singleton subjects. $\boldsymbol{F}$, Developmental changes in network-level intersubject variability during the first year of life. $\mathbf{G}$, Developmental changes in network-level intersubject variability during the second year of life. Dashed lines for $\mathbf{C}-\mathbf{G}$ correspond with the mean $(\boldsymbol{C}-\boldsymbol{E}) /$ mean changes $(\boldsymbol{F}, \boldsymbol{G})$ of intersubject variability of all voxels in the brain. Error bars represent SEM of voxels within network masks.

olds, which is highly consistent with its moderate variability observed in adults (Mueller et al., 2013). Another interesting pattern was that the auditory/language network showed the second lowest variability in neonates but climbed in ranking during development (Fig. 1C-E). Developmentally, voxels within all networks collectively showed significant decreases in variability during the first year, whereas voxels within all networks showed significant increases in variability during the second year (Fig. $1 F, G)$. The raw value maps (i.e., without mean-centering) of the intersubject variability for singletons, DZ, and $\mathrm{MZ}$ twins are presented in Figure 2, which depicts a highly consistent pattern across singleton and twin groups and the developmental changes in the raw magnitude of intersubject variability were more apparent.

The relationships between variability and short/long-range connectivity are shown for each age group in Figure 3. Consistent with previous findings in adults reporting a positive correlation between intersubject variability and percentage distant connectivity $(r=0.32, p<0.001)$ and a negative correlation between intersubject variability and degree local connectivity $(r=-0.33$, $p<0.001$; Mueller et al., 2013), we observed qualitatively similar patterns in all three infant groups. However, age-dependent development in such relationships was again apparent. Particularly, neonates demonstrated a weak, yet statistically significant positive correlation between intersubject variability and percentage distant connectivity ( $r=0.05, p<0.001)$, although this relationship became much stronger and comparable to that in adults in both 1- and 2-year-olds ( $r=0.36, p<0.001$ for 1-year-olds; $r=$ $0.31, p<0.001$ for 2-year-olds). Similarly, for the relationship between intersubject variability and local connectivity degree, neonates showed weaker negative correlations $(r=-0.14, p<$ $0.001)$ than 1 -year-olds $(r=-0.31, p<0.001)$, and 2-year-olds $(r=-0.34, p<0.001)$. To further validate these findings, we repeated these analyses using two additional connectivity thresholds ( $r=0.15$ and $r=0.35$ ), as well as two additional distance thresholds for both local and distant connectivity (age-groupspecific radii reported above $\pm 4 \mathrm{~mm}$, altogether 27 combinations for the 3 parameters). Alterations to each of these parameters resulted in a highly consistent pattern with the reported results. Specifically, both relationships were in the same direction and remained significant for all iterations for both 1- and 2-year-olds. In neonates, the relationships between intersubject variability and local connectivity (negative correlations) remained in the same direction and were significant for all iterations, whereas for percentage distant connectivity, 26/27(96\%) were in the same direction and 23/27 (85\%) were significant.

To examine the genetic influences on the brain's functional connectivity, the voxelwise intersubject variability of DZ and MZ twin pairs (Fig. 2) were normalized against those of the singleton pairs and similarly visualized on brain surfaces (Fig. $4 A$ ). Intriguingly, there was a trend of decreasing intersubject variability (cooler colors) with increasing genetic sharing magnitude (i.e., $\mathrm{SZ}>\mathrm{DZ}>\mathrm{MZ}$ ) among widespread brain regions across all three age groups, strongly suggesting the existence of genetic effects on the brain's intrinsic functional connectivity. To quantify these effects, GLM modeling was used to produce whole brain voxelwise genetic coefficient maps, which are shown in Figure $4 B$ with the areas demonstrating significant genetic/environmental effects presented in Figure $4 C, D$, respectively $(p<0.05$ after clusterwise multiple-comparisons correction). The spatial distribution of significant genetic effects was nonuniform across age groups (Fig. 4C). During the neonatal period, genetic effects were present in the posterior cingulate cortex, cuneus, fusiform gyrus, and bilateral middle and inferior frontal gyri. Consistently, at the network level, the three visual networks and the right frontoparietal network demonstrated above-average effects of heritability at this age (Fig. 4E). For 1-year-olds, genetic effects were found in the paracentral lobule, posterior cingulate cortex, bilateral lingual gyrus, right middle occipital gyrus, right angular gyrus/ parietal-temporal-occipital junction, bilateral middle and inferior 
frontal gyri, and ventromedial prefrontal cortex. At the network level, the higherorder cognitive networks (i.e., bilateral frontoparietal networks and the salience network) and two visual networks (occipital pole network and lateral visual network) demonstrated above-average effects of heritability in 1-year-olds (Fig. $4 E$ ). In 2-year-olds, genetic effects were most apparent in the left middle temporal gyrus and left supramarginal gyrus (Fig. $4 E$ ); consistently, the auditory network ranked highest in genetic effects at this age group. Compared with genetic effects, few significant environmental effects were observed across all three age groups (Fig. $4 D$ ). Developmentally, moderate networklevel (across all involved voxels) increases of genetic effects were observed during the first year ( 6 of 9 networks showed statistically significant increases in genetic effects), whereas significant network-level decreases dominated the second year (all 9 networks showed statistically significant decreases in genetic effects).

The local neighborhood-based correlation pattern between intersubject variability and genetic effects is presented in Figure 5. The overall patterns across each age group suggested that higher genetic influence was associated with both higher and lower intersubject variability in an age- and region-specific manner. Specifically, in neonates, regions in the right posterior cingulate cortex, anterior cingulate cortex, bilateral anterior insula, and bilateral precentral gyrus showed a positive relationship. Other regions exhibited negative correlations, particularly across lateral frontal regions, middle cingulate cortex, paracentral lobule, thalamus, bilateral posterior insula, left posterior middle temporal gyrus, and right supramarginal gyrus. For 1-year-olds, positive gene-variability correlations were found in regions including the anterior cingulate cortex, left medial frontal gyrus, bilateral caudate, thalamus, left precentral gyrus, bilateral middle occipital gyrus, and posterior cingulate cortex. On the other hand, major negative correlations were observed in the ventromedial prefrontal cortex, bilateral middle and inferior frontal gyrus, right posterior insula, bilateral postcentral gyrus, paracentral lobule, and precuneus. Two-year-olds exhibited positive gene-variability correlations in the bilateral insula, thalamus, rostral anterior cingulate cortex, middle and posterior cingulate cortex, bilateral middle occipital gyrus, right middle temporal gyrus, and bilateral precentral gyrus. Negative correlations were found in the dorsal anterior cingulate cortex, right anterior middle frontal gyrus, inferior and superior parietal lobules, and right superior temporal gyrus. Similar distribution patterns were

\section{Singletons}

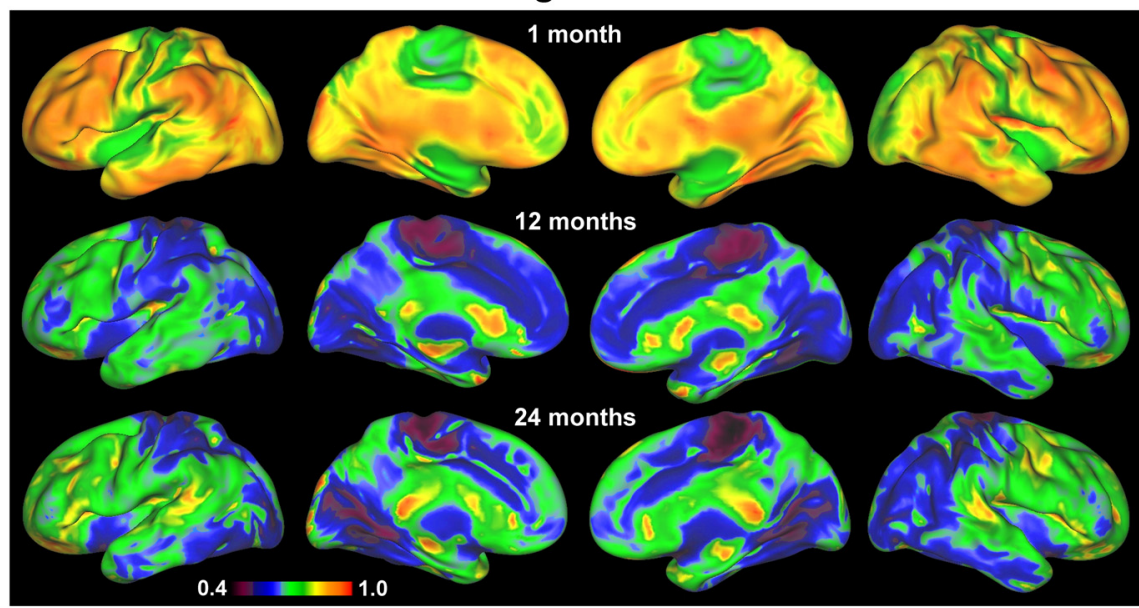

Dizygotic Twins

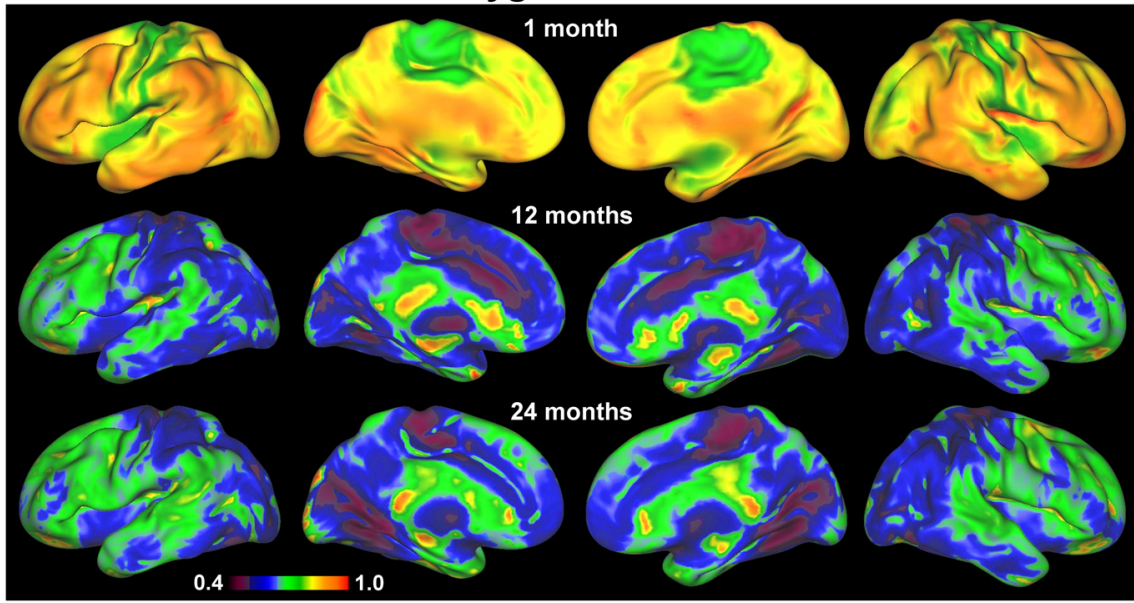

Monozygotic Twins

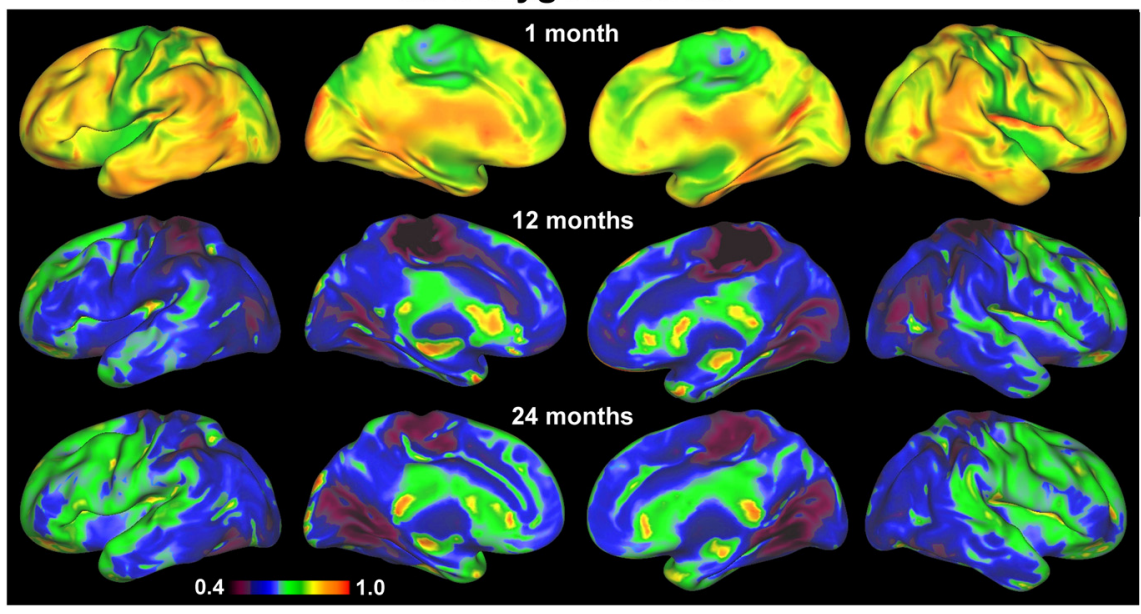

Figure 2. Variability maps (not demeaned) of singletons, DZ twins, and MZ twins for the three age groups.

observed when assessed at different neighborhood sizes (i.e., 7 and 57 voxels).

\section{Discussion}

In this study, the intersubject variability of and the genetic effects on the brain's functional connectivity during infancy were delineated. First, a general pattern of lower intersubject variability in primary functional areas and higher variability in association ar- 

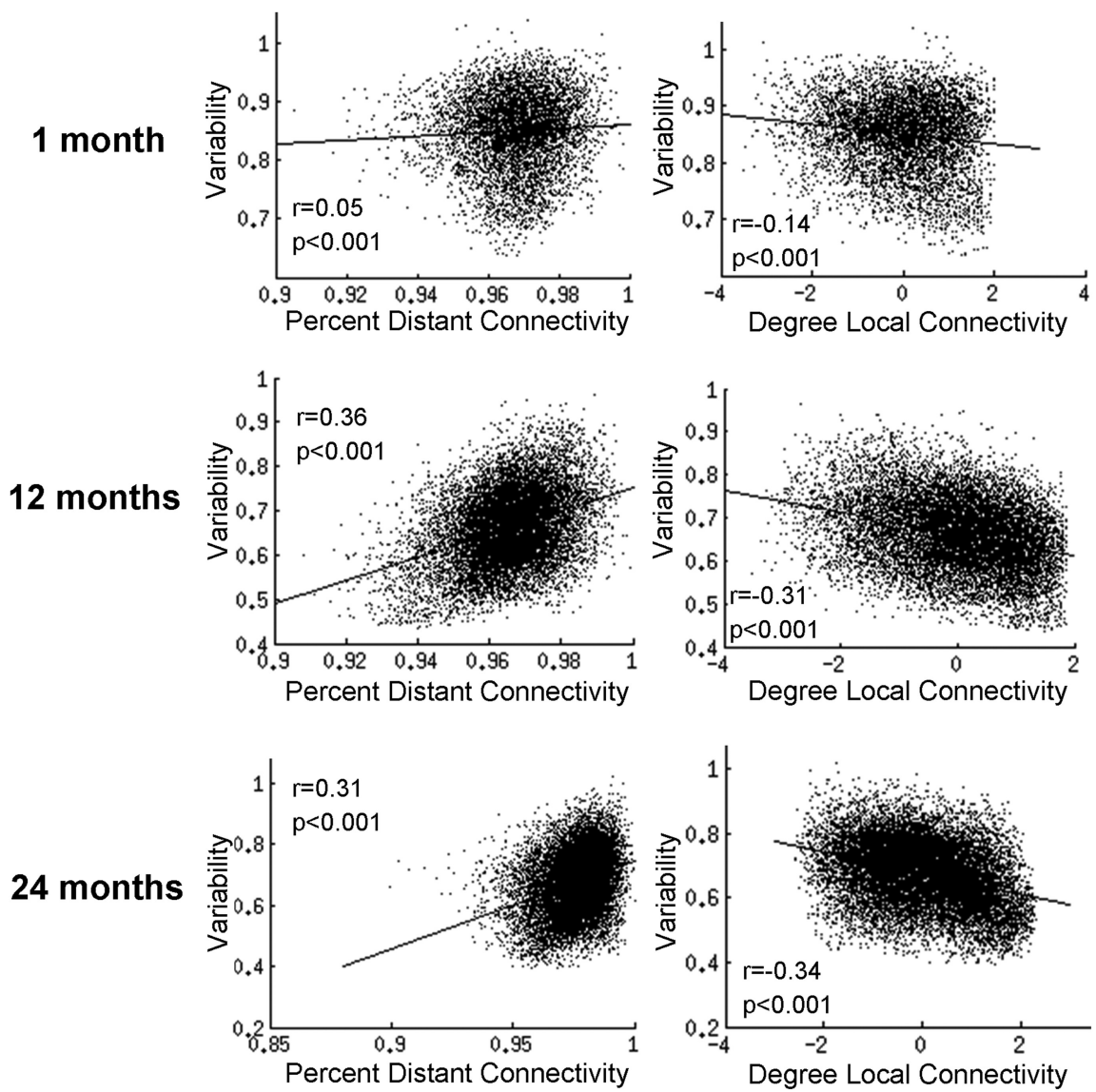

Figure 3. Relationships between intersubject variability of the brain's functional connectivity and distant/local connectivity during infancy.

eas resembling that of adults (Mueller et al., 2013) emerged in neonates and persisted during the first 2 years of life, suggesting the foundation for individual differences in the brain is established early in life. However, infant-specific patterns were also observed. Second, the magnitude of intersubject variability underwent a dramatic decrease during the first year, whereas the second year was characterized by moderate increases, thus showing an interesting U-shape. Thirdly, an age- and region-specific expression of genetic effects was detected. Finally, the relationship between genetic effects and intersubject variability of the brain's functional connectivity revealed interesting agedependent bidirectional patterns suggesting the existence of a rather complex relationship during infancy.

\section{The spatial distribution of intersubject variability during infancy}

The finding of a pattern of intersubject variability with many qualitative similarities as adults (Mueller et al., 2013) during the first 2 years of life is intriguing (Figs. 1,2). Mueller et al. (2013) postulated that protracted maturation and weaker genetic influence may be possible reasons for the higher variability in association areas observed in adults. By examining the earliest period of postnatal brain development and incorporating both singleton and twin subjects to directly assess the genetic effects, our study provided a unique opportunity to examine such hypotheses. First, Mueller et al. (2013) suggest that the relatively prolonged maturation process of association areas provides a wider window for environmental factors to show their effects during development, which might contribute to the higher variability in associ- ation areas observed in adults. Results in this study are generally consistent with this hypothesis but the underlying mechanism of environmental contributions might differ since higher variability in widespread association areas was observed in neonates with relatively limited postnatal environmental exposure. Actually, the prolonged maturation of higher order functional networks itself may largely explain the observed pattern. Specifically, previous studies have consistently documented that primary networks are largely established in neonates (Lin et al., 2008; Smyser et al., 2010; Doria et al., 2010), whereas high-order cognitive networks experience prolonged synchronization (Gao et al., 2009a, 2011, 2013; Tau and Peterson, 2010). Therefore, the earlier establishment of primary networks may yield a more consistent functional architecture across individuals leading to the observed lower intersubject variability in neonates. On the other hand, the immature structure, and possibly the varying pace of development, of higher-order networks may explain their higher intersubject variability in neonates.

Despite the generally consistent trend with adults, there are apparent infantspecific patterns. For example, the medial prefrontal/anterior cingulate areas demonstrate lower variability consistently across the three age groups, which is in contrast with their higher variability observed in adults (Fig. $1 A$; Mueller et al., 2013). This observation may be explained by the "skill learning" development theory in that these areas may govern lower level functions with lower variability early in life but with development they may progressively become more specialized and differentiated as more complex cognitive skills are acquired (Johnson, 2011). Consistent with this notion, the early establishment of the auditory network for the early functioning of auditory functions (Hykin et al., 1999) may help explain its initial low intersubject variability in neonates; the possible shift toward language-related higher order functions later in life may explain the observed increasing ranking in variability with age (Fig. $1 B-D$ ). Additionally, the subcortical and insula regions are also among areas showing lower variability during infancy. The fact that subcortical areas develop earlier than cortical regions (Tau and Peterson, 2010) and the insula is the earliest developing structure, both structurally (Afif et al., 2007) and functionally (Gao et al., 2011; Alcauter et al., 2013), implies that earlier development of their corresponding circuits may underlie their observed lower intersubject variability.

\section{The temporal development of intersubject variability during infancy}

Temporally, the general decrease of intersubject variability observed during the first year of life (Fig. $1 B$ ) is interesting. The well documented dramatic development of the brain's various functional networks (Johnson, 1994; Gao et al., 2009a, 2011, 2012; Tau and Peterson, 2010; Alcauter et al., 2013), and the concurrent robust development of gray and white matter (Mukherjee et al., 


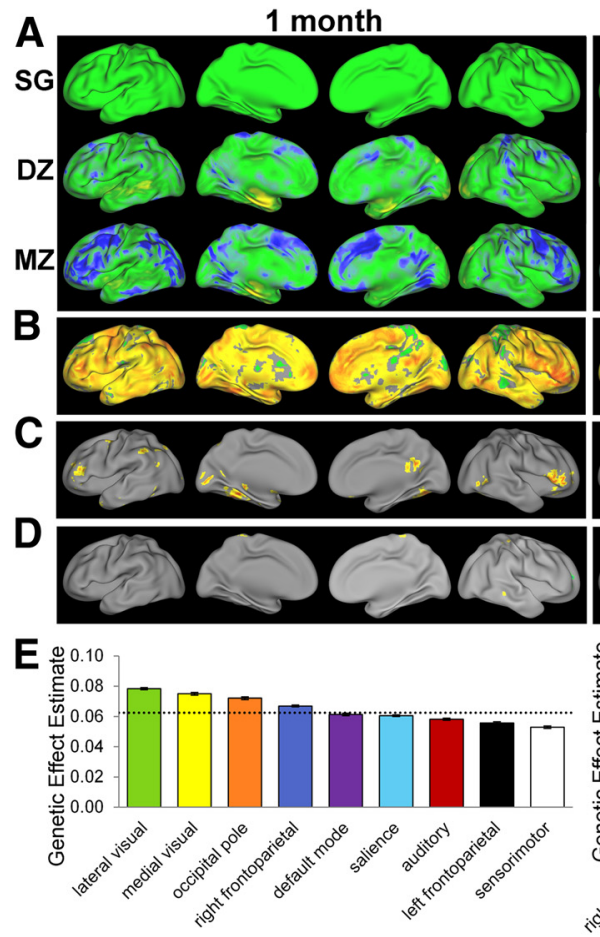

$\mathbf{F}$ 誉

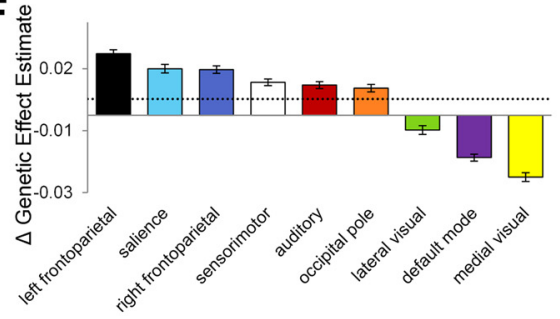

12 months
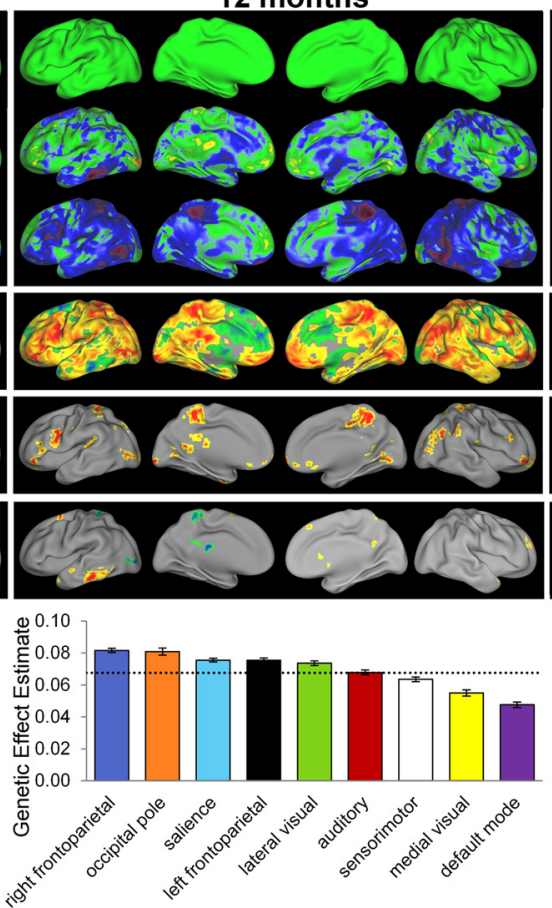

G

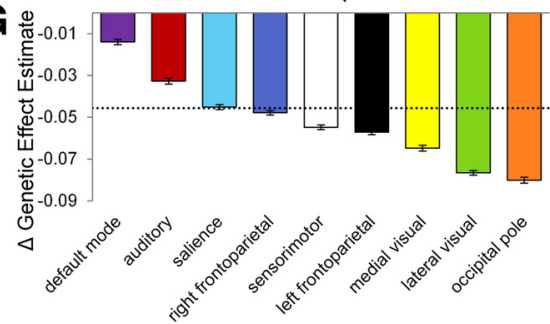

Figure 4. Genetic effects on the brain's functional connectivity during infancy. A, Comparison of the intersubject variability the brain's functional connectivity between singleton pairs (SG, top row), DZ twin (middle row), MZ twin (bottom row) pairs. The values of DZ and MZ pairs were normalized against those of the singleton pairs to show their relative strength. $B$, Genetic coefficient maps based on GLM modeling of the genetic effects on the brain's functional connectivity. $C$, Areas showing statistically significant genetic effects $(p<0.05$ after cluster-wise multiple-comparisons correction) in each of the three age groups. $\boldsymbol{D}$, Areas showing statistically significant effects of shared environment ( $p<0.05$ after cluster-wise multiple-comparisons correction) in each of the three age groups. $\boldsymbol{E}$, Relative ranking of different functional networks in genetic effects for all three age groups. $\boldsymbol{F}$, Developmental changes in network-level genetic effects during the first year of life. $\boldsymbol{G}$ Developmental changes in network-level genetic effects during the second year of life. Dashed lines in $\boldsymbol{E}-\boldsymbol{G}$ correspond with the mean $(\boldsymbol{E}) /$ mean genetic changes $(\boldsymbol{F}, \boldsymbol{G})$ of all networks. Error bars represent SEM.

2002; Schneider et al., 2004; Gao et al., 2009b; Geng et al., 2012) during this period suggest that the first year of life may be characterized by the establishment of the "backbone" structures of most functional networks, which likely possess high degree of commonalities across individuals. Therefore, this "backbone" construction process may help explain the observed global decrease in variability during this period. However, subsequent development of the brain circuits may include more activity-dependent refinement processes, which are likely more subject-specific (Johnson, 2000, 2011). Consequently, the pattern of decreasing variability may reverse due to the increasing contribution of subject-specific and experience-based refinement processes. Indeed, our results show a moderate but consistent recovery of intersubject vari-

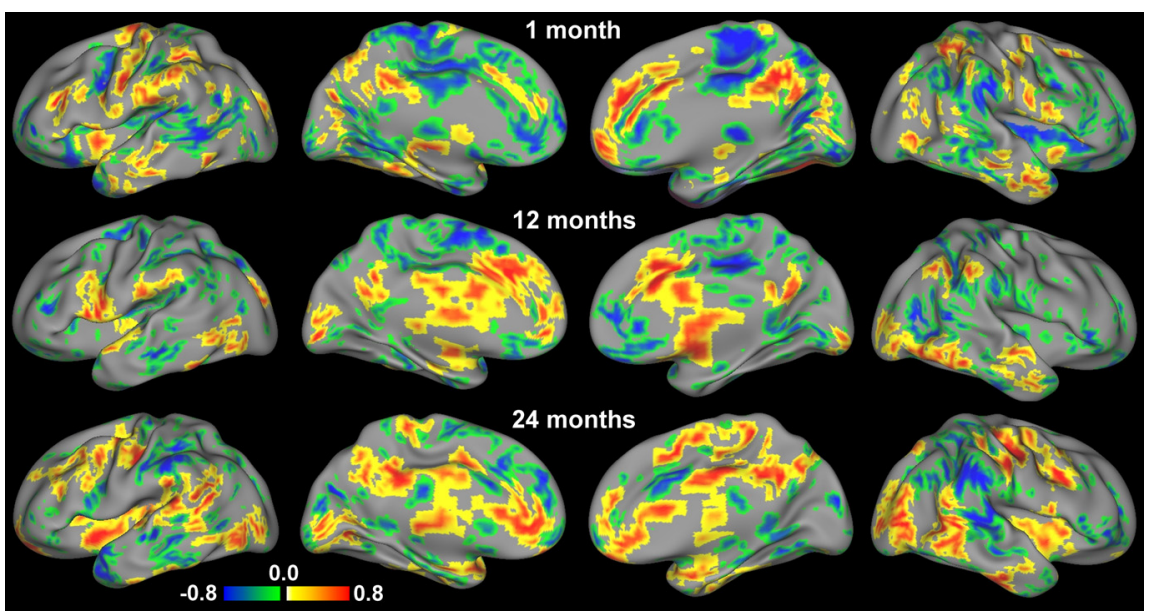

Figure 5. Relationship between the intersubject variability of and genetic effects on the brain's functional connectivity. Areas with statistically significant ( $p<0.05$ after multiple-comparison correction) positive (red) and negative (blue) correlations between intersubject variability and genetic effects are visualized for the three age groups. 
ability during the second year of life (Fig. $1 B, G$ ). Regarding the relationship between variability and connection distance, we have observed a generally adult-like pattern characterized by positive correlations between variability and percentage distant connectivity but negative correlations between variability and degree local connectivity. However, the strength of both the positive and negative correlations show dramatic improvement during development, especially between neonates and 1-year-olds during which time both values are comparable with adults (Fig. 2). These findings indicate that robust associations between long/short range connectivity and intersubject variability are established during postnatal development and likely arise as a result of the improved functioning status of these connections.

\section{Genetic effects on the intersubject variability during infancy}

Mueller et al. (2013) postulated that weaker genetic control may contribute to the higher intersubject variability in association areas. However, findings in this study challenge this view and show that there is not a clear distinction between primary and association areas in the relationship between intersubject variability and genetic influences during infancy. For example, although effects of heritability were observed in visual cortex in neonates and 1-year-olds, higher-order frontal and parietal cortices also demonstrated significant genetic effects (Fig. $4 B, C$ ). At network level, different categories of networks are intermixed in their genetic effect rankings (Fig. $4 E$ ). In fact, a direct analysis of the correlation between these two measures (Fig. 5) showed that genetic effects could be related to both higher and lower intersubject variability in a region- and age-specific manner. Therefore, our findings indicate that both commonality and uniqueness in our brain's wiring scheme could be preserved across generations through genetic heritage. Evolutionally, this preserved coexistence of similarity and variability may be essential to maintain both species-related stability and adaptiveness for the everchanging external environment (Sporns and Edelman, 1993). From a developmental trajectory perspective, our results also do not support a simplistic inverse relationship. For example, during the first year, we observed a massive decrease in intersubject variability across the whole brain (Fig. $1 B$ ) but there were only moderate increases in genetic expression during this period (Fig. $4 F$ ). In contrast, the more modest increase of variability during the second year is accompanied by more dramatic decrease in genetic effects expression (Figs. 1B, 4G). Overall, our findings point to an age-dependent and region-specific relationship between intersubject variability and genetic contributions during infancy whose developmental implications deserve more investigation.

\section{Limitations}

Several potential limitations of this study deserve discussion. First, it is possible that different sleep stages across subjects may induce bias to our results. However, in our protocol, all subjects were imaged as soon as they fell asleep to reduce such variability. Moreover, previous studies (Vincent et al., 2007; Horovitz et al., 2008) have provided evidence regarding the limited effects of sleep on resting BOLD so it is likely that such effects are minimal in the proposed study. Second, there might be infant-specific regions in different networks that are not present in their adult configurations so the adoption of adult networks as references likely ignored such regions. Future studies are needed to further explore this aspect. In addition, despite the state-of-the-art algorithms used for image registration, warping of infant brain data to an adult template is an inherently challenging task. Thus, there could be minor errors introduced in the registration process and future efforts are needed to further improve this procedure. Finally, in our pairing of the singletons, factors including race, socioeconomic status, and ethnicity were not controlled and their effects in the variability calculation need further investigation.

\section{Conclusions}

Our results indicate that key properties of the brain's functional connectivity, including both its intersubject variability and genetic effects, show intriguing spatial distributions and temporal dynamics during infancy. For intersubject variability, adult-like features characterized by lower values in primary functional areas and higher values in association areas are evident starting from birth. However, infant-specific and age-dependent developmental patterns are also apparent. Temporally, an interesting U-shape development is observed. For genetic effects, nonuniform spatial distributions and bidirectional relationships with intersubject variability were observed, challenging the simplistic, unidirectional view that weaker genetic control is related to higher variability. Overall, the individual variability of and the genetic effects on the brain's functional organization during infancy delineated in this study may provide new perspectives for future investigations of different developmental disorders.

\section{References}

Afif A, Bouvier R, Buenerd A, Trouillas J, Mertens P (2007) Development of the human fetal insular cortex: study of the gyration from 13 to 28 gestational weeks. Brain Struct Funct 212:335-346. CrossRef Medline

Alcauter S, Lin W, Smith JK, Gilmore JH, Gao W (2013) Consistent anterior-posterior segregation of the insula during the first two years of life. Cereb Cortex, in press. CrossRef Medline

Biswal B, Yetkin FZ, Haughton VM, Hyde JS (1995) Functional connectivity in the motor cortex of resting human brain using echo-planar MRI. Magn Reson Med 34:537-541. CrossRef Medline

Cox RW (1996) AFNI: software for analysis and visualization of functional magnetic resonance neuroimages. Comput Biomed Res 29:162-173. CrossRef Medline

Doria V, Beckmann CF, Arichi T, Merchant N, Groppo M, Turkheimer FE, Counsell SJ, Murgasova M, Aljabar P, Nunes RG, Larkman DJ, Rees G, Edwards AD (2010) Emergence of resting state networks in the preterm human brain. Proc Natl Acad Sci U S A 107:20015-20020. CrossRef Medline

Fransson P, Skiöld B, Horsch S, Nordell A, Blennow M, Lagercrantz H, Aden U (2007) Resting-state networks in the infant brain. Proc Natl Acad Sci U S A 104:15531-15536. CrossRef Medline

Gao W, Zhu H, Giovanello KS, Smith JK, Shen D, Gilmore JH, Lin W (2009a) Evidence on the emergence of the brain's default network from 2-weekold to 2-year-old healthy pediatric subjects. Proc Natl Acad Sci U S A 106:6790-6795. CrossRef Medline

Gao W, Lin W, Chen Y, Gerig G, Smith JK, Jewells V, Gilmore JH (2009b) Temporal and spatial development of axonal maturation and myelination of white matter in the developing brain. AJNR Am J Neuroradiol 30:290296. CrossRef Medline

Gao W, Zhu H, Giovanello K, Lin W (2010) Multivariate network-level approach to detect interactions between large-scale functional systems. Med Image Comput Comput Assist Interv 13:298-305. Medline

Gao W, Gilmore JH, Giovanello KS, Smith JK, Shen D, Zhu H, Lin W (2011) Temporal and spatial evolution of brain network topology during the first two years of life. PLoS One 6:e25278. CrossRef Medline

Gao W, Gilmore JH, Shen D, Smith JK, Zhu H, Lin W (2013) The synchronization within and interaction between the default and dorsal attention networks in early infancy. Cereb Cortex 23:594-603. CrossRef Medline

Gao W, Alcauter S, Smith J, Gilmore JWL (2014a) Development of human brain cortical network architecture during infancy. Brain Struct Funct, in press. CrossRef Medline

Gao W, Alcauter S, Elton A, Hernandez-Castillo C, Smith J, Ramirez J, Lin W (2014b) Functional network development during the first year: relative sequence and socioeconomic correlations. Cereb Cortex, in press. CrossRef Medline 
Geng X, Gouttard S, Sharma A, Gu H, Styner M, Lin W, Gerig G, Gilmore JH (2012) Quantitative tract-based white matter development from birth to age 2years. Neuroimage 61:542-557. CrossRef Medline

Gilmore JH, Lin W, Prastawa MW, Looney CB, Vetsa YS, Knickmeyer RC, Evans DD, Smith JK, Hamer RM, Lieberman JA, Gerig G (2007) Regional gray matter growth, sexual dimorphism, and cerebral asymmetry in the neonatal brain. J Neurosci 27:1255-1260. CrossRef Medline

Gilmore JH, Shi F, Woolson SL, Knickmeyer RC, Short SJ, Lin W, Zhu H, Hamer RM, Styner M, Shen D (2012) Longitudinal development of cortical and subcortical gray matter from birth to 2 years. Cereb Cortex 22:2478-2485. CrossRef Medline

Horovitz SG, Fukunaga M, de Zwart JA, van Gelderen P, Fulton SC, Balkin TJ, Duyn JH (2008) Low frequency BOLD fluctuations during resting wakefulness and light sleep: a simultaneous EEG-fMRI study. Hum Brain Mapp 29:671-682. CrossRef Medline

Hykin J, Moore R, Duncan K, Clare S, Baker P, Johnson I, Bowtell R, Mansfield P, Gowland P (1999) Fetal brain activity demonstrated by functional magnetic resonance imaging. Lancet 354:645-646. CrossRef Medline

Johnson MH (1994) Visual attention and the control of eye movements in early infancy. In: Attention and performance XV: conscious and nonconscious information processing (Umilta C, Moscovitch M, eds), pp 291310. Cambridge, MA: MIT.

Johnson MH (2000) Functional brain development in infants: elements of an interactive specialization framework. Child Dev 71:75-81. CrossRef Medline

Johnson MH (2011) Interactive specialization: a domain-general framework for human functional brain development? Dev Cogn Neurosci 1:721. CrossRef Medline

Kriegeskorte N, Goebel R, Bandettini P (2006) Information-based functional brain mapping. Proc Natl Acad Sci U S A 103:3863-3868. CrossRef Medline

Lin W, Zhu Q, Gao W, Chen Y, Toh CH, Styner M, Gerig G, Smith JK, Biswal B, Gilmore JH (2008) Functional connectivity MR imaging reveals cortical functional connectivity in the developing brain. AJNR Am J Neuroradiol 29:1883-1889. CrossRef Medline

McIntosh AR (2000) Towards a network theory of cognition. Neural Netw 13:861-870. CrossRef Medline

Mueller S, Wang D, Fox MD, Yeo BT, Sepulcre J, Sabuncu MR, Shafee R, Lu J, Liu H (2013) Individual variability in functional connectivity architecture of the human brain. Neuron 77:586-595. CrossRef Medline

Mukherjee P, Miller JH, Shimony JS, Philip JV, Nehra D, Snyder AZ, Conturo TE, Neil JJ, McKinstry RC (2002) Diffusion-tensor MR imaging of gray and white matter development during normal human brain maturation. AJNR Am J Neuroradiol 23:1445-1456. Medline

Power JD, Barnes KA, Snyder AZ, Schlaggar BL, Petersen SE (2012) Spurious but systematic correlations in functional connectivity MRI networks arise from subject motion. Neuroimage 59:2142-2154. CrossRef Medline Power JD, Barnes KA, Snyder AZ, Schlaggar BL, Petersen SE (2013) Steps toward optimizing motion artifact removal in functional connectivity MRI: a reply to Carp. Neuroimage 76:439-441. CrossRef Medline

Schneider JF, Il'yasov KA, Hennig J, Martin E (2004) Fast quantitative diffusion-tensor imaging of cerebral white matter from the neonatal period to adolescence. Neuroradiology 46:258-266. CrossRef Medline

Sepulcre J, Liu H, Talukdar T, Martincorena I, Yeo BT, Buckner RL (2010) The organization of local and distant functional connectivity in the human brain. PLoS Comput Biol 6:e1000808. CrossRef Medline

Seung S (2013) Connectome: how the brain's wiring makes us who we are. New York: Houghton Mifflin Harcourt

Shen D, Davatzikos C (2004) Measuring temporal morphological changes robustly in brain MR images via 4-dimensional template warping. Neuroimage 21:1508-1517. CrossRef Medline

Shi F, Yap PT, Wu G, Jia H, Gilmore JH, Lin W, Shen D (2011) Infant brain atlases from neonates to 1- and 2-year-olds. PLoS One 6:e18746. CrossRef Medline

Shi F, Wang L, Dai Y, Gilmore JH, Lin W, Shen D (2012) LABEL: pediatric brain extraction using learning-based meta-algorithm. Neuroimage 62 : 1975-1986. CrossRef Medline

Smith SM, Jenkinson M, Woolrich MW, Beckmann CF, Behrens TE, Johansen-Berg H, Bannister PR, De Luca M, Drobnjak I, Flitney DE, Niazy RK, Saunders J, Vickers J, Zhang Y, De Stefano N, Brady JM, Matthews PM (2004) Advances in functional and structural MR image analysis and implementation as FSL. Neuroimage 23:S208-219. CrossRef Medline

Smith SM, Fox PT, Miller KL, Glahn DC, Fox PM, Mackay CE, Filippini N, Watkins KE, Toro R, Laird AR, Beckmann CF (2009) Correspondence of the brain's functional architecture during activation and rest. Proc Natl Acad Sci U S A 106:13040-13045. CrossRef Medline

Smyser CD, Inder TE, Shimony JS, Hill JE, Degnan AJ, Snyder AZ, Neil JJ (2010) Longitudinal analysis of neural network development in preterm infants. Cereb Cortex 20:2852-2862. CrossRef Medline

Smyser CD, Snyder AZ, Neil JJ (2011) Functional connectivity MRI in infants: exploration of the functional organization of the developing brain. Neuroimage 56:1437-1452. CrossRef Medline

Snyder K, Webb S, Nelson C (2002) Theoretical and methodological implications of variability in infant brain response during a recognition memory paradigm. Infant Behav Dev 25:466-494. CrossRef

Sporns O, Edelman GM (1993) Solving Bernstein's problem: a proposal for the development of coordinated movement by selection. Child Development 64:960-981. CrossRef Medline

Tau GZ, Peterson BS (2010) Normal development of brain circuits. Neuropsychopharmacology 35:147-168. CrossRef Medline

Vincent JL, Patel GH, Fox MD, Snyder AZ, Baker JT, Van Essen DC, Zempel JM, Snyder LH, Corbetta M, Raichle ME (2007) Intrinsic functional architecture in the anaesthetized monkey brain. Nature 447:83-86. CrossRef Medline 